\title{
Electrophysiological and Behavioral Evidence Demonstrating That Predator Detection Alters Adaptive Behaviors in the Snail Lymnaea
}

\author{
Michael V. Orr and Ken Lukowiak \\ Hotchkiss Brain Institute, Faculty of Medicine, University of Calgary, Calgary, Alberta, Canada T2N 4N1
}

\begin{abstract}
Stress has been shown to both impair and enhance learning, long-term memory (LTM) formation, and/or its recall. The pond snail, Lymnaea stagnalis, both detects and responds to the scent of a crayfish predator with multiple stress-related behavioral responses. Using both behavioral and electrophysiological evidence, this investigation is a first attempt to characterize how an environmentally relevant stressor (scent of a predator) enhances LTM formation in Lymnaea. Using a training procedure that, in "standard" pond water (PW), results in an intermediate-term memory that persists for only $3 \mathrm{~h}$, we found that training snails in "crayfish effluent" (CE) induces a memory that persists for $48 \mathrm{~h}$ (i.e., its now an LTM). In addition, if we use a training procedure that in PW produces an LTM that persists for $1 \mathrm{~d}$, we find that snails trained in CE have an LTM that persists for at least $8 \mathrm{~d}$. Furthermore, we describe how a single neuron (RPeD1), which has been shown to be a necessary site for LTM formation, reflects the behavioral changes in its firing properties that persist for the duration of the LTM. Finally, Lymnaea exhibit context-specific memory, that is, when a memory is formed in a specific context (food odorant), it is only recalled in that context. Here, we found that snails trained in CE demonstrate context generalization, that is, memory is recalled in multiple contexts. All data are consistent with the hypothesis that learning in a stressful, yet biologically relevant, environment enhances LTM and prolongs its retention.
\end{abstract}

Key words: fear; predation; Lymnaea; long-term memory; crayfish; operant conditioning

\section{Introduction}

Gaining understanding into the behavioral responses of prey to the presence of their predators, at both the behavioral and cellular level, is of great interest to both neurobiological and ecological disciplines. We demonstrated that the pond snail Lymnaea stagnalis both detects and responds to the scent of a crayfish predator with multiple stress-related behavioral responses (Orr et al., 2007). These predator-induced behavioral changes are reflected as altered electrophysiological properties in the neuron RPeD1, which is responsible for initiating the aerial respiratory behavior (Orr et al., 2007). Here we test the hypothesis that predator detection will enhance long-term memory (LTM) formation assayed at both the behavioral and neurophysiological level.

In aquatic environments, chemicals cues are important signals between and among species (Burks and Lodge, 2002). Lymnaea responds to predators by using avoidance behaviors such as a withdrawal response when under attack (Rigby and Jokela, 2000), crawling above the water line (Dalesman et al., 2006) and increasing sensitivity to the shadow-elicited withdrawal response when crayfish effluent (CE) is detected (Orr et al., 2007).

Received Nov. 19, 2007; revised Jan. 31, 2008; accepted Feb. 1, 2008.

This work was supported by Canadian Institutes of Health Research. We thank Dr. Louise Page, Hyo-jung Orr, David Rosenegger, Kara Martens, Kashif Parvez, and Kim Browning for all their help and comments.

Correspondence should be addressed to Ken Lukowiak, Hotchkiss Brain Institute, Faculty of Medicine, University of Calgary, Calgary, Alberta, Canada T2N 4N1. E-mail: lukowiak@ucalgary.ca.

DOI:10.1523/JNEUROSCI.5132-07.2008

Copyright $\odot 2008$ Society for Neuroscience $\quad$ 0270-6474/08/282726-09\$15.00/0
Aerial respiratory behavior in Lymnaea is driven by a threeneuron network whose sufficiency and necessity has been experimentally demonstrated (Syed et al., 1990, 1992). This behavior can be operantly conditioned to induce memory formation (Lukowiak et al., 1996, 1998; McComb et al., 2005). The duration of the memory is dependent on the training procedure used. A single 30 min training session (TS) results in intermediate-term memory (ITM) that persists for up to $3 \mathrm{~h}$, whereas two 30-min training sessions with a $1 \mathrm{~h}$ rest interval results in LTM that persists for $24 \mathrm{~h}$ (Lukowiak et al., 1998, 2000, 2003b; Parvez et al., 2006). ITM is dependent on new protein synthesis, whereas LTM is dependent on both new protein synthesis and altered gene activity (Sangha et al., 2003a). Neural correlates of LTM have been demonstrated in one of the neurons that drives aerial respiratory behavior, RPeD1 (Spencer et al., 1999, 2002; McComb et al., 2005). More importantly, we have further demonstrated that the molecular changes necessary for LTM formation, reconsolidation, extinction, and forgetting, occur within RPeD1 (Scheibenstock et al., 2002; Sangha et al., 2003a,b).

LTM in Lymnaea is context specific, that is, snails trained in the presence of a food odor only demonstrate memory when tested in the same food odor (Haney and Lukowiak, 2001). When learning during a stressful event, the context of that event forms an intrinsic part of the situation to be remembered (de Kloet et al., 1999; Haney and Lukowiak, 2001; Joels et al., 2006).

Here we demonstrate that, when Lymnaea are faced with an ecologically relevant stressor, the "smell" of a crayfish predator, 
newly formed memories are both significantly increased and are context generalized. This is the first demonstration we know of in which interspecies chemical communication augments LTM formation in an organism in which the molecular events in a single neuron have been shown to be necessary for LTM formation.

\section{Materials and Methods}

Snails. Lymnaea stagnalis were reared in the snail facility at the University of Calgary from a strain of Lymnaea originally obtained from Vrije Universeit in Amsterdam. The ancestors of these snails were obtained from canals in a polder located near Utrecht, The Netherlands in the early 1950s. Cohorts of 10-14 adult snails with a shell length of $21-26 \mathrm{~mm}$ were labeled and maintained within home aquaria at room temperature $\left(\sim 20^{\circ} \mathrm{C}\right)$ until training.

Brief description of aerial respiratory behavior. Lymnaea are bimodal breathers obtaining oxygen through either cutaneous respiration (i.e., directly through their skin) or through aerial respiration via a rudimentary lung [i.e., gas exchange with the atmosphere (Lukowiak et al., 1996, 1998)]. To perform aerial respiration, the snail must surface and open its pneumostome (the respiratory orifice) while contracting and relaxing the appropriate respiratory muscles. Thus, training snails not to perform aerial respiration is not harmful. For a more detailed description, see Lukowiak et al. (2003a,b).

Operant conditioning. Snails were removed from home aquaria and placed into a $1 \mathrm{~L}$ beaker containing $500 \mathrm{ml}$ of hypoxic dechlorinated water [i.e., pond water $(\mathrm{PW})]$ at an ambient temperature of $\sim 20^{\circ} \mathrm{C}$. The pond water is made hypoxic by bubbling $\mathrm{N}_{2}$ gas through the water for 20 min before introducing the snails. The animals are given a $10 \mathrm{~min}$ acclimatization period before the $30 \mathrm{~min}$ training session. By subjecting snails to a hypoxic challenge, the animals increase their rate of aerial respiration (Lukowiak et al., 1996). The animals are operantly conditioned by applying a gentle tactile stimulus with a sharpened wooden applicator to their pneumostome on pneumostome opening. The stimulus is strong enough to cause the snails to close the pneumostome yet gentle enough that the snails do not perform the full-body withdrawal response. This pneumostome closer response is a graded part of the whole-snail escape response (Inoue et al., 1996). Every time the snail opens its pneumostome and receives the stimulus during the training period, the time is recorded for future use in the yoked control experiments. Yoked controls (see below) were performed for all behavioral and electrophysiological experiments. All behavioral experiments were done "blind" in which the person performing the training paradigm was unaware of the status of the cohort being tested.

Two training paradigms were used: (1) The ITM-training procedure and (2) The LTM-training procedure. The ITM procedure consists of a single $30 \mathrm{~min}$ TS, after which the snails are returned to their home aquaria. The snails are then tested for memory (TM) (i.e., a "savings test") using a similar test to that of the training session or the group is then subject to electrophysiological testing in lieu of the TM. The time of the TM or recording is at the indicated times after the TS. The LTMtraining procedure consists of two $30 \mathrm{~min}$ training sessions separated by a $1 \mathrm{~h}$ interval. The snails are placed into a eumoxic holding tank for the $1 \mathrm{~h}$ interval between TS1 and TS2 and after TS2. The TM or electrophysiological recording is performed at the indicated times after TS2.

Yoked control experiments. Snails that received yoked training were treated in an identical manner as that outlined above (see Operant conditioning), with one exception (Lukowiak et al., 1996, 1998, 2000, 2003b). During the training period, yoked snails received exactly the same number of stimuli using the same pattern of stimulation as those of the operant conditioning group; however, the stimuli were not contingent on pneumostome opening. However, these snails did receive a contingent stimulus to the pneumostome during the savings test session.

Exposure to crayfish and crayfish effluent. Cambrus spp. are housed in a $110 \mathrm{~L}$ aquarium and maintained on a diet of snails and lettuce. We trained snails as above only using $500 \mathrm{ml}$ of water taken from the crayfish aquaria. We termed this water CE (Orr et al., 2007). During the CE training procedures, snails are not trained in the presence of crayfish, only water taken from aquaria containing crayfish. Both the ITM- and
LTM-training procedures are identical to that described above save the use of CE.

We also sought to determine whether direct exposure to predators had any effect on learning and memory. Therefore, during direct predator exposure experiments, snails were directly exposed to the crayfish predator for a period of $2 \mathrm{~h}$. Snails were placed directly in the crayfish tank containing two crayfish that had not been fed for $24 \mathrm{~h}$. Snails that survived the direct exposure with minimal or no damage to their shell were removed and immediately placed in a $500 \mathrm{ml}$ beaker containing CE and subject to the ITM-training procedures as described above.

Semi-intact preparation and electrophysiological recordings. The preparations were dissected similar to that described previously (Spencer et al., 1999, 2002; Inoue et al., 2001; McComb et al., 2003, 2005), except that only the penis was removed and the head/foot complex and buccal mass were left fully intact. Preparations were pinned down in individual recording dishes with their dorsal sides uppermost. The central ring ganglia (CNS) were pinned to the dish directly through the foot musculature dorsal-side up. The outer sheath surrounding the CNS was removed using fine forceps. Standard electrophysiological techniques were used as described previously in Lymnaea semi-intact preparations. Intracellular recordings were obtained using sharp glass microelectrodes filled with saturated $\mathrm{K}_{2} \mathrm{SO}_{4}$ solution. Tip resistances of the microelectrodes used for recordings ranged from 20 to $30 \mathrm{M} \Omega$. Intracellular signals were amplified via a Neurodata Instruments (New York, NY) amplifier and displayed simultaneously on a Macintosh (Apple Computers, Cupertino, CA) PowerLab/-4SP (ADInstruments, Castle Hill, New South Wales, Australia) and a Hitachi (Tokyo, Japan) oscilloscope. Recordings were analyzed and stored using the PowerLab software. For complete details, see McComb et al. $(2003,2005)$. Once RPeD1 was successfully impaled, the cells were given a minimum 10-min stabilization period, after which a $600 \mathrm{~s}$ trace was used for analysis. Ten electrophysiological characteristics were measured for each recording and are as follows: (1) total number of action potentials (APs) per $600 \mathrm{~s}$, (2) total frequency, (3) resting membrane potential, (4) number of APs per burst, (5) burst frequency, (6) after hyperpolarization of the first AP in each burst, (7) average AP peak of each burst, (8) AP rise time, (9) AP fall time, and (10) burst duration.

Operational definition of learning and memory. As described previously (Lukowiak et al., 1996, 1998, 2000) for ITM-training, memory is defined as a significant reduction in the number of pneumostome openings after training. That is, TM must be significantly less than TS1. In the case of LTM-training, TS2 must be significantly lower than TS1 and TM must not be significantly greater that TS2.

Feeding behavior. We used the well described and robust feeding behavior of Lymnaea (Benjamin et al., 2000) as a positive control for our change of context experiments. To observe the feeding behavior, individual snails were placed in a $15 \mathrm{ml}$ Petri dish on a clear Plexiglas stand raised $10 \mathrm{~cm}$ above a mirror. The dish was filled with pond water, carrot water ( $14 \mathrm{ml}$ of pond water and $1 \mathrm{ml}$ of freshly ground and filtered carrot juice), crayfish water, or cray/carrot water (14 ml of crayfish water and $1 \mathrm{ml}$ of carrot juice), respectively. The feeding behavior was measured for each snail by averaging the number of rasps per minute (rpm) for three 1-min periods in each context. Between each trial, the snails were rinsed and placed in standard pond water for $2 \mathrm{~h}$. Half of the snails were given the treatments in the order presented here, and half of the snails were given the treatments in reverse order to that described here.

Statistics. We analyzed within-group water treatment effects on snail behavioral data with repeated-measures ANOVA in which the withinsubject factors of TS1, TS2, and X hours/days-TM when appropriate and the between-subject factor of interval (time in hours or days) were used. All repeated-measures data were tested for equal variance using Mauchlys test for sphericity. In cases in which sphericity could not be assumed, we used the conservative adjusted Greenhouse-Geisser $p$ values. For cases in which we identified a significant interaction between the repeated factor and water treatment, we used repeated contrasts to identify which treatment pairs differed significantly. Between-group factors (comparing separate cohorts) were analyzed using one-way ANOVA with Tukey's post hoc test to detect cases in which we identified a significant interaction. Electrophysiological data were analyzed using ANOVA also with Tukey's post hoc test to detect cases in which we identified a 
significant interaction. Nonhomogenous data (number of spikes per $600 \mathrm{~s}$ interval etc.) were log transformed to homogenize between treatment data before ANOVA. Context feeding behavior observations were analyzed using a paired-sample $t$ test for each combination of factors. In all analysis reported here, a type I error rate of 0.05 was used. All statistics were run on SPSS (Chicago, IL) Mac OSX version 11.0.4.

\section{Results}

\section{The $24 \mathrm{~h}$ post-CE exposure experiments}

We reported recently (Orr et al., 2007) that exposure of a snail to $\mathrm{CE}$ for $2 \mathrm{~h}$ immediately before dissection results in a significant reduction in the spontaneous firing frequency and number of spikes per burst in RPeD1. To assess the effect of CE and operant conditioning on the behavior of snails and the electrophysiological characteristics of RPeD1, we had to first determine how long the effects of CE exposure persisted on both snail behavior and $\mathrm{RPeD} 1$ activity in the absence of operant conditioning training. We therefore exposed snails to CE for $2 \mathrm{~h}$, after which we then placed them back into their home aquaria for $24 \mathrm{~h}$. Snails were then given either the behavioral test (a single 30 min training session, i.e., ITM-training) or were dissected, and electrophysiological recordings made from $\mathrm{RPeD} 1$. We found that snails given the ITM-training $24 \mathrm{~h}$ after CE exposure demonstrated ITM that lasted for $3 \mathrm{~h}$ but did not demonstrate LTM (Fig. $1 \mathrm{~A}$ ). That is, the number of attempted pneumostome openings during the TM was significantly different from TS1 after $3 \mathrm{~h}$ but not after $24 \mathrm{~h}$, confirming previous data (Lukowiak et al., 2000; Parvez et al., $2005,2006)$. Yoked controls also did not demonstrate a difference in the number of pneumostome openings between TS1 and the TM. Furthermore, we found that, $24 \mathrm{~h}$ after CE exposure, the electrophysiological characteristics of RPeD1 were similar to that of CE-naive snails. That is, there were no significant differences in spontaneous firing frequency, bursting frequency, and number of spikes/burst between naive snails and snails exposed to CE $24 \mathrm{~h}$ before (Fig. $1 B$ ). To ensure that exposure to $\mathrm{CE}$ alone does not result in long-term sensitization of aerial respiratory behavior, we compared the number of attempted pneumostome openings during TS1 $24 \mathrm{~h}$ after the exposure to CE compared with the number of attempted openings during operant conditioning in PW alone or in CE during an initial exposure. We found that there was no significant difference between these three values $(n=22, p>0.05)$ (data not shown but can be inferred from the TS1 bars in Figs. 1, 2) We conclude that exposure to CE alone has only transitory, short-lived (i.e., $<24$ h) effects on snail behavior and the electrophysiological phenotype of RPeD1.

\section{ITM-training procedure experiments}

\section{Exposure to $C E$}

To determine whether exposure to a predator "scent" altered the snails' ability to form memory compared with training in PW alone, we first examined what effect, if any, training in CE would have on memory formation when snails were subjected to the ITM-training procedure. First, two cohorts of naive snails were given ITM-training in PW and then tested at either 3 or $24 \mathrm{~h}$ later. As already demonstrated (Lukowiak et al., 2000, 2003a,b; Taylor and Lukowiak, 2000; Parvez et al., 2006), these snails demonstrated memory at $3 \mathrm{~h}$ but did not exhibit memory when tested $24 \mathrm{~h}$ later. That is, the ITM-training procedure resulted in an intermediate-term memory, but no significant difference was found between the TS and the $24 \mathrm{~h}$ TM, indicating that no LTM was formed (Fig. $2 A$, black bars). We next asked whether training snails in CE would result in augmented memory, and, if so, how long would the memory persist? To answer this question, new
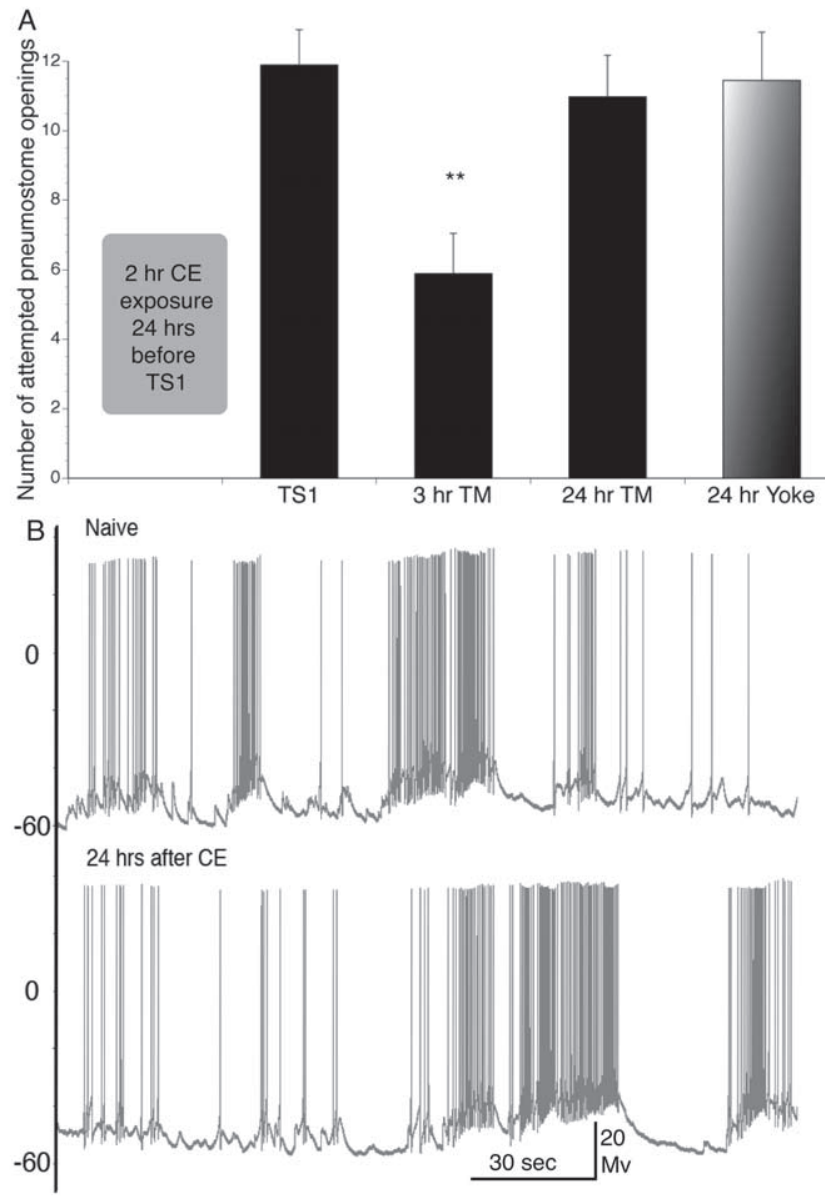

Figure 1. A, Operant conditioning of aerial respiratory behavior using the ITM-training procedure in PW $24 \mathrm{~h}$ after a $2 \mathrm{~h}$ CE exposure. Snails demonstrate ITM $3 \mathrm{~h}$ after operant conditioning of aerial respiratory behavior but do not demonstrate memory $24 \mathrm{~h}$ after. A significant reduction in the number of attempted pneumostome openings was observed after $3 \mathrm{~h}$ but not in the $24 \mathrm{~h}$ test for memory (24h TM) or in the yoked controls compared with TS1 ( $3 \mathrm{~h} \mathrm{TM}, n=18, p<$ $0.01 ; 24 \mathrm{~h} \mathrm{TM}, n=22, p>0.05 ;$ yoke, $n=20, p>0.05)$. B , Representative electrophysiological recordings from RPeD1 in an untrained semi-intact preparation obtained $24 \mathrm{~h}$ after the intact snails were exposed to either PW (Naive, top) or CE (24 hrs after CE, bottom). No significant differences were found between naive and CE-exposed RPeD1s in any of the 10 measured electrophysiological parameters ( $n=9 ; p>0.05$ in all analyses; data not shown).

cohorts of snails were given ITM-training in CE water and tested for memory (in PW) 24, 48, and $72 \mathrm{~h}$ later. Yoked controls were also performed in CE and tested in PW. To our amazement, snails only exposed to CE during TS1 exhibited LTM when tested 24 and $48 \mathrm{~h}$ later but not at $72 \mathrm{~h}$ (Fig. $2 \mathrm{~A}$, white bars). That is, the mean number of attempted pneumostome openings was significantly decreased in the 24 and $48 \mathrm{~h} \mathrm{TM}$ but not the $72 \mathrm{~h}$ TM compared with TS1. In yoked control experiments in CE (Fig. $2 A$, faded bars), there was no statistical difference between TS and yoked TM. The number of attempted pneumostome openings at the 24 and $48 \mathrm{~h}$ TM was also significantly lower than the yoked controls at these time periods and significantly lower than the $72 \mathrm{~h} \mathrm{TM}$ in CE. Thus, we conclude that a single $30 \mathrm{~min}$ training session in CE is sufficient to cause LTM formation that persists for at least $48 \mathrm{~h}$.

Knowing that exposure to $\mathrm{CE}$ alone produces an effect on RPeD1 that lasts $2 \mathrm{~h}$ (Orr et al. 2007) but not $24 \mathrm{~h}$ (Fig. 1), we next asked whether the electrophysiological profile of RPeD1 in snails subject to an ITM paradigm in CE was also altered. To answer 

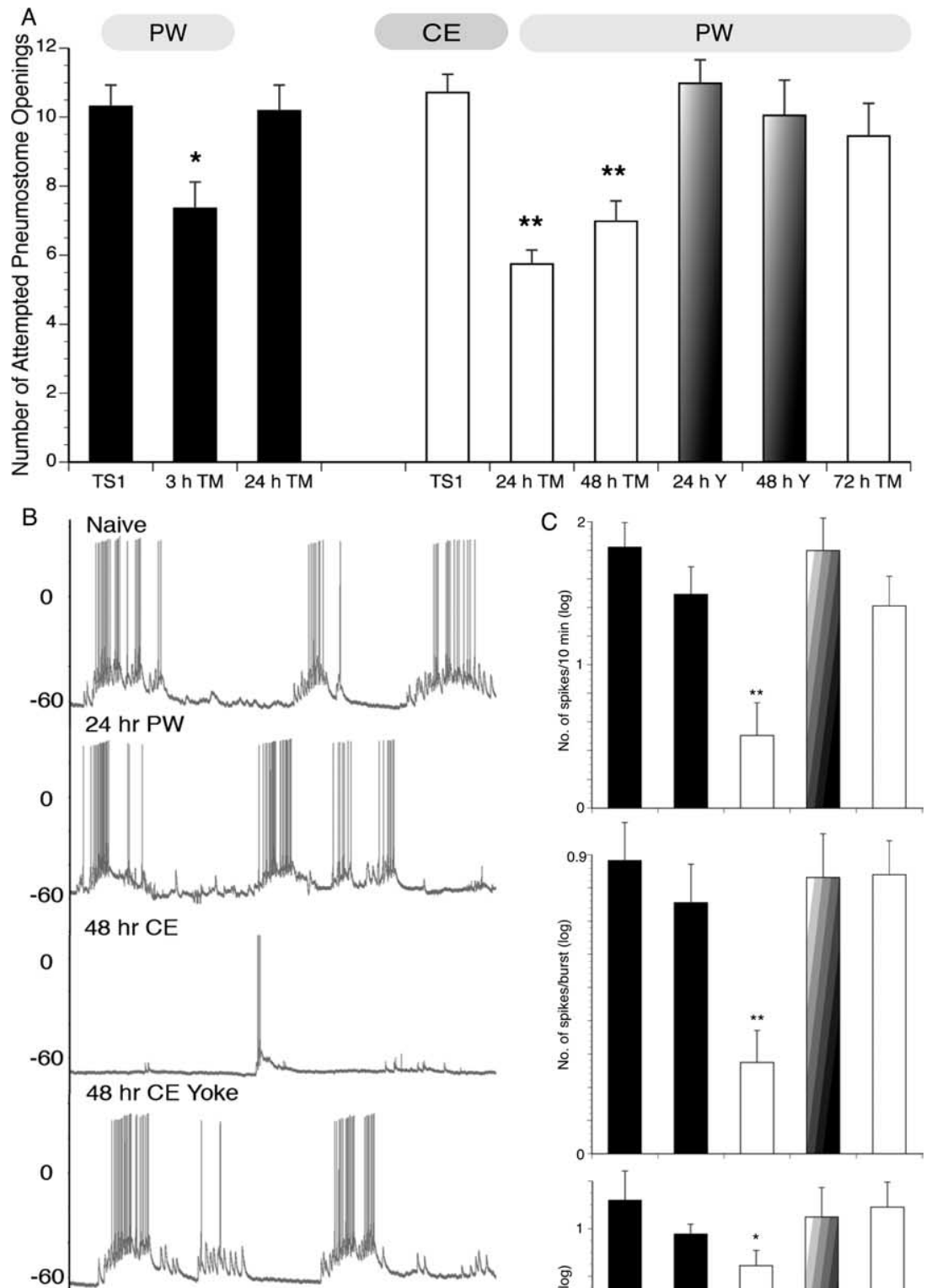
$72 \mathrm{hr} C \mathrm{E}$
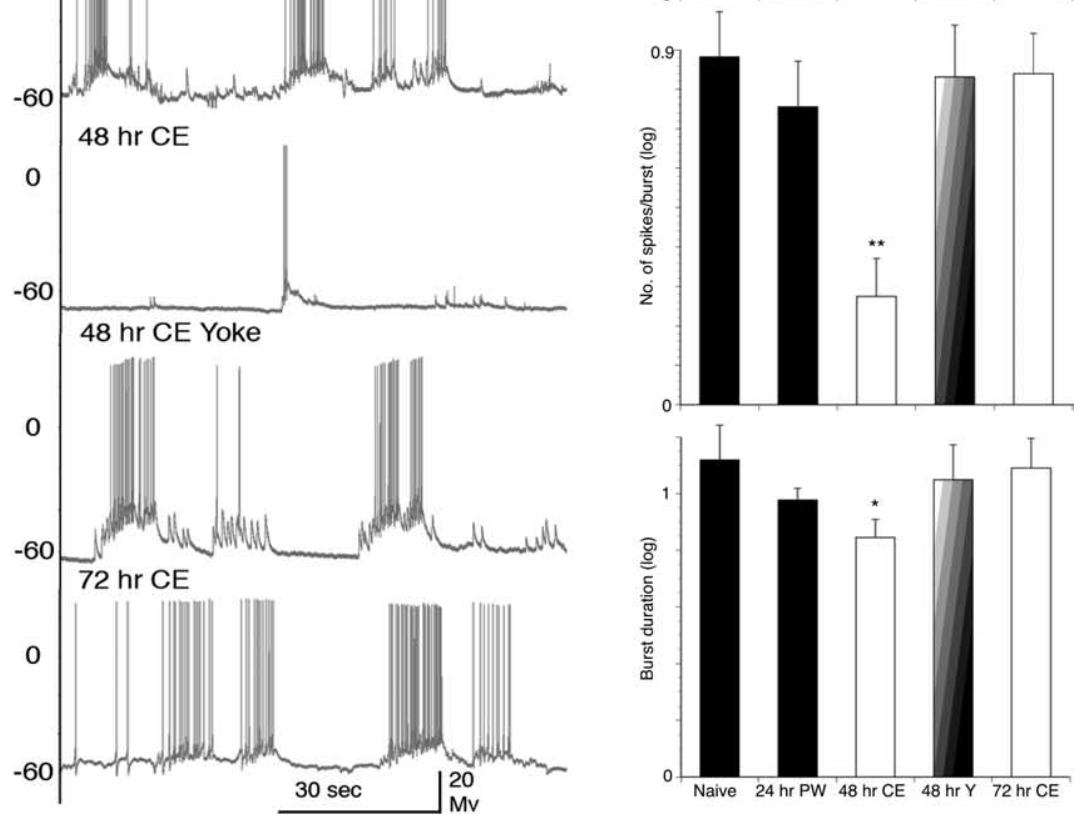

Figure 2. Behavioral data and representative electrophysiological recordings of intact Lymnaea and RPeD1 from semi-intact animals after the ITM-training procedure in either PW or CE. $\boldsymbol{A}$, The ITM-training procedure in PW results in intermediate-term memory but does not result in LTM (i.e., a memory lasting $24 \mathrm{~h} ; 3 \mathrm{~h} \mathrm{TM}, n=18, p<0.05 ; 24 \mathrm{~h} \mathrm{TM}, n=44, p>0.05$; blackbars). However, the ITM-training procedure in CEresults in LTM. That is, the number of attempted pneumostome openings is significantly lower than TS1 and time-matched controls (i.e., memory at 24 and $48 \mathrm{~h}$; white bars; $24 \mathrm{~h} \mathrm{TM}, n=35, p<0.01$; $48 \mathrm{~h} \mathrm{TM}, n=41, p<0.01$ ). Snails did not demonstrate memory formation in the 24 or 48 h yoked control groups (faded bars; $n=30, p>0.05$ and $n=20, p>0.05$ for 24 and 48 h yoked, respectively) or $72 \mathrm{~h}$ after training (white bar; $n=22 ; p>0.05$ ). $\boldsymbol{B}$, Representative recordings from RPeD1 in the naive state (untrained and in PW), $24 \mathrm{~h}$ after PW-ITM-training, $48 \mathrm{~h}$ after ITM-training procedure in (E, 48 h CE-ITM yoke control, and $72 \mathrm{~h}$ after the ITM-training procedure in CE. C, Summary data for mean \pm SEM spiking activity/600 s and number of spikes per burst (top and middle bar graphs, respectively; values log transformed). Results for the $48 \mathrm{~h} \mathrm{ITM-training} \mathrm{procedure} \mathrm{in} \mathrm{CE} \mathrm{are} \mathrm{significantly} \mathrm{lower} \mathrm{than} \mathrm{the} \mathrm{naive} \mathrm{state} \mathrm{(} n=7$; $p<0.01$ ). Results for $24 \mathrm{~h}$ ITM-training procedure in PW-trained animals, $48 \mathrm{~h} \mathrm{ITM}$-training procedure in CE-yoked, and $72 \mathrm{~h} \mathrm{ITM}$-training procedure in CE-trained animals are not significantly different from the naive state ( $24 \mathrm{~h} \mathrm{PW}$-ITM, $n=8, p>0.05 ; 48 \mathrm{~h}$ CE-ITM yoked, $n=8, p>0.05 ; 72 \mathrm{hCE}-\mathrm{ITM}, n=6, p>0.05$ ). Bottom bar graph demonstrates summary data for burst duration (mean \pm SEM, values log transformed) of each treatment. ITM-training procedure in CE at $48 \mathrm{~h}$ are significantly lower than the naive state $(n=7 ; p<0.05)$. ITM-training procedure in PW at $24 \mathrm{~h}$, ITM-training procedure in CE at $48 \mathrm{~h}$ yoked, and ITM-training procedure in CE at $72 \mathrm{~h}$ are not significantly different from the naivestate (24h PW-ITM, $n=8, p>0.05 ; 48 \mathrm{hCE}-\mathrm{ITMyoked}, n=8, p>0.05 ; 72 \mathrm{hCE}-\mathrm{ITM}, n=6, p>$ 0.05). No significant differences were detected between treatments in other electrophysiological parameters measured (see Materials and Methods). this, we first needed to determine the electrophysiological properties of RPeD1 in snails given ITM-training in PW. Thus, a new cohort of snails were given the ITMtraining procedure in $\mathrm{PW}$, and then, $24 \mathrm{~h}$ later, these snails were dissected and recordings were made from RPeD1. There were no significant differences in the measured characteristics of RPeD1 in the $24 \mathrm{~h}$ recordings from the ITM-trained group compared with recordings taken from naive snails (Fig. $2 B$, top two traces, $C$, first two bars in each graph).

We then gave the same ITM-training procedure to a new group of snails, but instead of using PW, the snails were trained in CE. We knew from our behavioral experiments that training in $\mathrm{CE}$ induced robust memory for at least $48 \mathrm{~h}$. We therefore examined the properties of CEITM-trained RPeD1 after 48 and $72 \mathrm{~h}$. As in the behavioral experiments, we also examined a yoked control group at $48 \mathrm{~h}$. We found that snails given ITM-training in CE demonstrated significantly reduced spontaneous firing frequency (measured by the number of spikes/600 s), the spikes/bursts, and the burst duration in the $48 \mathrm{~h}$ operantly conditioned group but not in the $48 \mathrm{~h}$ yoked or the $72 \mathrm{~h}$ operant groups (Fig. $2 B$, bottom three traces, $C$, right three bars). From these behavioral and electrophysiological data, we conclude exposure to CE during ITM-training produces LTM. These data also suggest that, for predator-induced enhancement of LTM, the electrophysiological changes in $\mathrm{RPeD} 1$ associated with these memories parallel the duration of the behavioral phenotype.

\section{LTM-training paradigm experiments}

Because training in CE had such a significant enhancing effect on memory formation in snails receiving the ITM-training procedure (a single $30 \mathrm{~min}$ training session), we wanted to see what effect the LTM-training procedure (i.e., two $30 \mathrm{~min}$ sessions separated by a $1 \mathrm{~h}$ interval) in CE would have on memory formation. A cohort of naive snails was given LTMtraining in PW (Fig. 3A, black bars). Snails demonstrated learning (TS2) and memory when tested 24 but not $48 \mathrm{~h}$ or $8 \mathrm{~d}$ later. Yoked controls did not demonstrate memory at $24 \mathrm{~h}$. Another cohort of naive snails was trained using the LTM-training procedure but this time in CE. Remarkably, these snails had an LTM that persisted for $8 \mathrm{~d}$ but not $10 \mathrm{~d}$ (Fig. 3A, white bars). That is, there is a reduction in the number of attempted pneumostome openings at $24 \mathrm{~h}$ and $8 \mathrm{~d}$ later, which were 
both significantly lower than the TS1 and the $8 \mathrm{~d}$ yoke and $10 \mathrm{~d}$ TM. CE-yoked control snails (faded bar) did not demonstrate memory at $8 \mathrm{~d}$. Thus, snails receiving LTM-training in CE had a significantly longer-lasting memory compared with snails trained in PW.

Next we compared the electrophysiological characteristics of RPeD1 in snails given the LTM-training procedure in PW and CE. We first measured the electrophysiological properties of RPeD1 in snails given the LTM-training procedure in PW after 24 and $48 \mathrm{~h}$, respectively. We found a significant reduction in the number of spikes/600 $s$ and the number of spikes per burst in RPeD1 from snails $24 \mathrm{~h}$ after the PW-LTM-training but not after $48 \mathrm{~h}$ (Fig. $3 B, C, 24 \mathrm{~h}$ PW and $48 \mathrm{~h} \mathrm{PW}$ ) compared with naive snail recordings. In contrast, we found that recordings from RPeD1 measured in snails given the CE-LTM-training demonstrated significant reductions in the number of spikes/600 s, number of spikes/ burst, and the burst duration after $8 \mathrm{~d}$ (Fig. $3 B, D, 8$ day $\mathrm{CE}$ ). These reductions in RPeD1 activity were not seen in the $8 \mathrm{~d}$ yoked or $10 \mathrm{~d}$ CE-LTM-treated RPeD1 recordings (Fig. $3 B, D, 8 \mathrm{~d}$ yoke and $10 \mathrm{~d}$ $\mathrm{CE})$. From these behavioral and electrophysiological data, we conclude the following: (1) that exposure to CE during LTM-training greatly extends the duration of LTM, and (2) that the electrophysiological changes in RPeD1 associated with these memories parallel the duration of the behavioral phenotype.

\section{Direct exposure to crayfish}

Considering our finding that snails exposed to water that has recently contained crayfish (i.e., CE) had such a profound effect on many stress-related behaviors (Orr et al., 2007) and augments newly formed memories to such a great extent, we asked the following question: does training and testing in CE further enhance LTM formation? In the data presented in Figure 2, training occurred in CE, but testing for memory took place in PW. We found that testing snails in CE did not alter the memory profile of memory duration (Fig. 4A).

Next we thought that, if snails had direct contact with the predator (i.e., were housed with and preyed on by crayfish), there might be an additional enhancement of LTM or possibly even a blockade of LTM formation. To test this hypothesis, a cohort of snails was placed directly in the crayfish tank for $2 \mathrm{~h}$ with two hungry crayfish. The surviving snails (crayfish typically consumed two to four snails) were then removed and subject to the ITM-
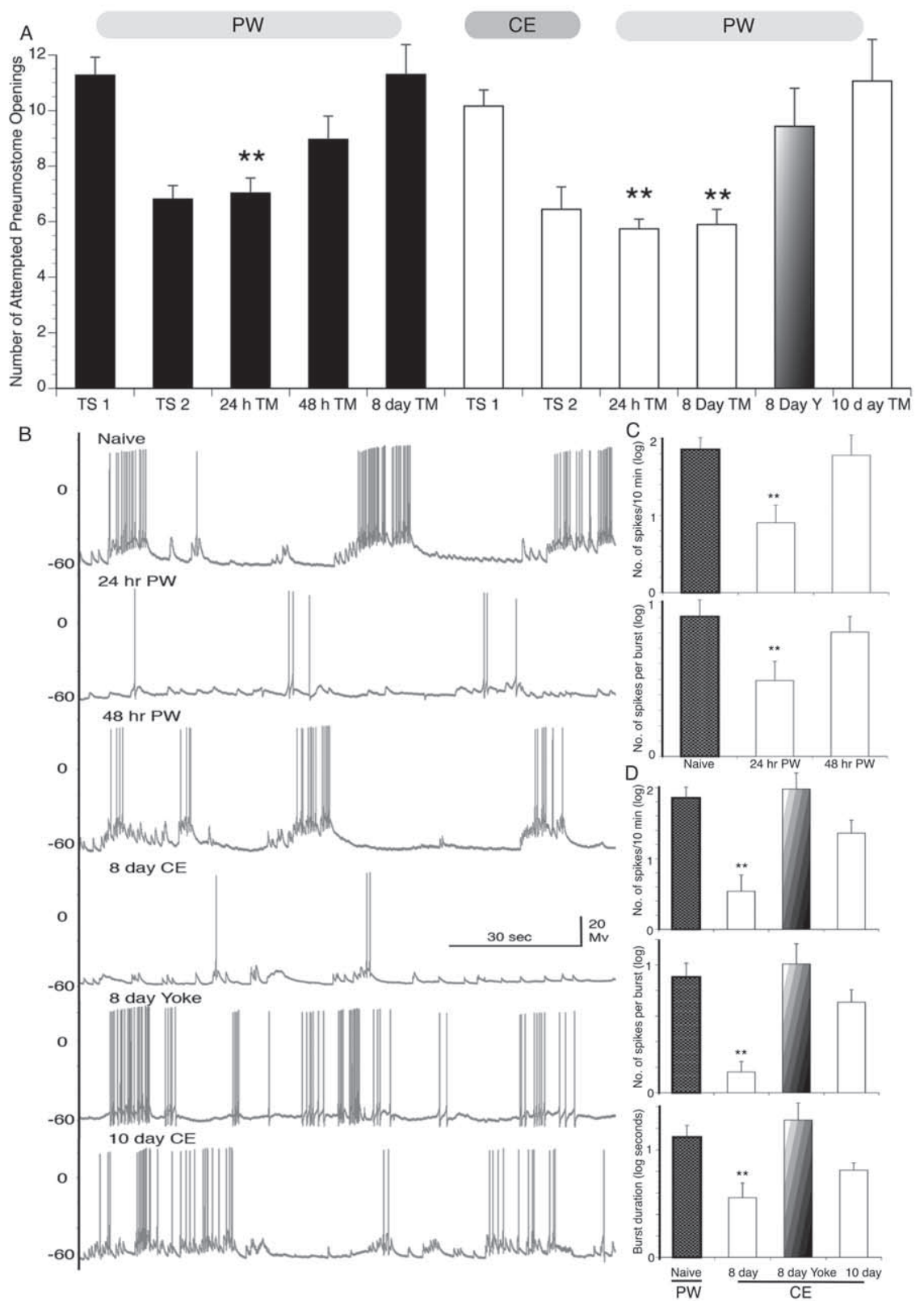

Figure 3. Behavioral data (intact snails) and representative electrophysiological recordings of RPeD1 from semi-intact preparations after the LTM-training procedure in either PW or CE. $A$, The LTM-training procedure in PW results in a memory that lasts 24 but not $48 \mathrm{~h}$ or $8 \mathrm{~d}$ (black bars; $24 \mathrm{~h} \mathrm{TM}, n=61, p<0.01 ; 48 \mathrm{~h} \mathrm{TM}, n=26, p>0.05 ; 8 \mathrm{~d}$ TM, $n=20, p>0.05$ ). However, the LTM-training procedure in CE results in a memory that persists for at least $8 \mathrm{~d}$. That is, the number of attempted pneumostome openings is significantly lower than TS1 and not significantly greater than TS2 as well as time-matched controls (white bars; $8 \mathrm{~d}$ TM, $n=29, p<0.01$ ). Snails did not demonstrate LTM in the $8 \mathrm{~d}$ yoked control groups (faded bars; $n=21, p>0.05$ ) nor was LTM present $10 \mathrm{~d}$ after the LTM-training procedure in CE (white bar; $n=16, p>0.05$ ). $\boldsymbol{B}$, Representative recordings from RPeD1 in a naive snail (untrained, PW), $24 \mathrm{~h}$ after the LTM-training procedure in PW, $48 \mathrm{~h}$ after the LTM-training procedure in PW, $8 \mathrm{~d}$ after the LTM-training procedure in CE, $8 \mathrm{~d}$ after the CE-yoke control, and $10 \mathrm{~d}$ after the LTM-training procedure in CE. C, Summary data for mean \pm SEM spiking activity/600 s and number of spikes per burst (top and bottom bar graph, respectively, values log transformed). The data $24 \mathrm{~h}$ after the LTM-training procedure in PW are significantly lower than the naive state $(n=7, p<0.01)$. However, the data $48 \mathrm{~h}$ after the LTM-training procedure in PW are not significantly different from the naive state $(n=7, p>$ 0.05). D, Summary data for mean \pm SEM spiking activity $/ 600$ s, number of spikes per burst, and burst duration (top, middle, and bottom bar graphs, respectively, values log transformed). The data $8 \mathrm{~d}$ after the LTM-training procedure in (E-trained animals are significantly lower than the naive state $(n=8, p<0.01)$ in all three measures. The data for the $8 \mathrm{~d}$ CE-yoked control and $10 \mathrm{~d}$ post-LTM-training procedure in animals are not significantly different from the naive state for all three measures ( $8 \mathrm{~d}$ CE-LTM yoked, $n=8, p>0.05 ; 10 \mathrm{~d}$ (E-LTM, $n=8, p>0.05)$. No significant differences were detected between treatments in other electrophysiological parameters measured (see Materials and Methods). 

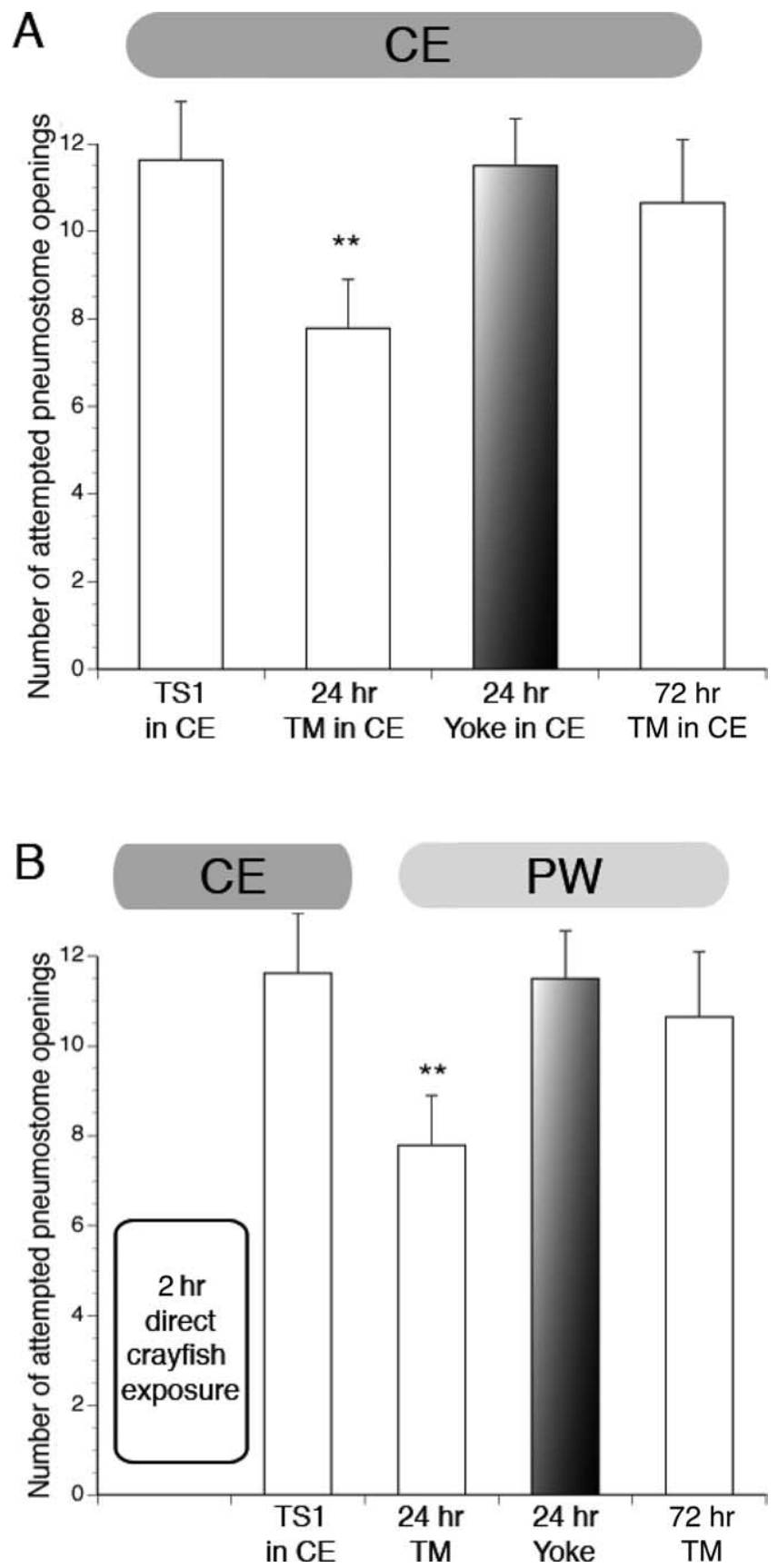

Figure 4. Memory profile of Lymnaea given the ITM-training procedure with testing for memory also in CE and after $2 \mathrm{~h}$ direct exposure to hungry crayfish predators. A, Animals trained and tested in CE demonstrated memory after $24 \mathrm{~h}$ but not $72 \mathrm{~h}$ or in $24 \mathrm{~h}$ yoked controls $(24 \mathrm{~h}$ TM, $n=20, p<0.01 ; 72 \mathrm{~h} \mathrm{TM}, n=17, p>0.05 ; 24$ h yoked, $n=19, p>0.05)$. $\boldsymbol{B}$, Animals trained in CE immediately after $2 \mathrm{~h}$ in direct contact with hungry crayfish demonstrated memory after $24 \mathrm{~h}$ but not $72 \mathrm{~h}$ ( $24 \mathrm{~h} \mathrm{TM}, n=24, p<0.01 ; 72 \mathrm{~h} \mathrm{TM}, n=20, p>0.05)$. Yoked control group did not demonstrate memory at $24 \mathrm{~h}$ ( $24 \mathrm{~h}$ yoked, $n=19, p>0.05$ ).

training paradigm in CE (Fig. $4 B$ ). We found that the memory profile was similar to snails trained in $\mathrm{CE}$ water alone (Fig. 2, compare $A$, white bars, with $B$ ) and not significantly different from snails both trained and tested in CE (Fig. $4 A$ ). That is, the memory persisted for 24 but not $72 \mathrm{~h}$. The yoked groups were similar to the yokes in the CE-only experiments in that the snails demonstrated no memory at $24 \mathrm{~h}$. We also compared the number of attempted pneumostome openings during the TS1 and $24 \mathrm{~h}$ TM between the directly exposed snails and the CE-only trained

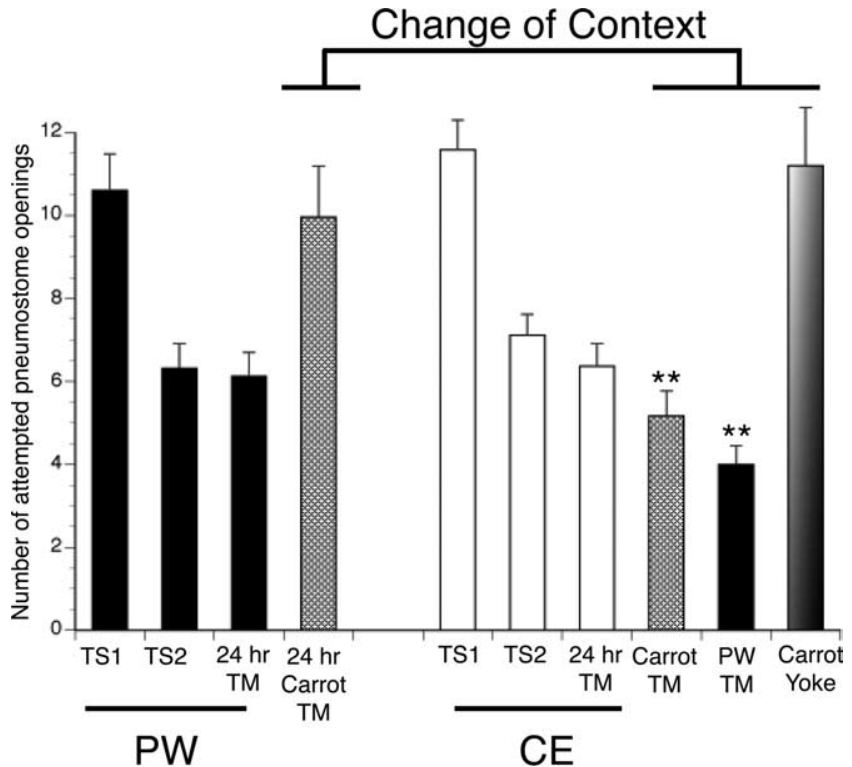

Figure 5. The effect of the LTM-training procedure in PW and CE on context-dependent LTM. Snails given the LTM-training procedure in PW demonstrate learning (TS2, $n=38, p<0.01$ ) and memory $24 \mathrm{~h}$ later ( $24 \mathrm{~h} \mathrm{TM}, n=38, p<0.01$; black bars); however, when challenged with a change of context test (i.e., carrot odor), they do not demonstrate memory (24 h carrot TM, $n=24, p>0.05$; hatched bar). Snails given the LTM-training procedure in (E demonstrate learning and memory (TS2, $n=36, p<0.01 ; 24 \mathrm{~h} \mathrm{TM}, n=36, p<0.01$ ). However, when challenged with a change of context test, carrot scent, or PW, memory continued to be observed (carrot TM, hatched bar, $n=28, p<0.01$; PW, black bar, $n=34, p<0.01$ ). That is, context generalization was observed. Carrot-yoked controls did not demonstrate memory (carrot yoke, $n=20, p>0.05$, faded bar).

snails and found that there was no significant difference between the number of openings for the comparable training session, respectively. That is, the TS1 sessions between the two cohorts were not significantly different, nor were the $24 \mathrm{~h}$ TM sessions. We therefore conclude that training and testing in CE or direct exposure to the predator did not augment memory beyond that of exposure to the predator scent only during training.

\section{Change of context experiments}

We demonstrated previously that memory formation in Lymnaea is context specific (Haney and Lukowiak, 2001). We therefore sought to test the hypothesis that exposure to predator scent (i.e., training in $\mathrm{CE}$ ) would alter the context specificity of newly formed memories. To test this hypothesis, snails were given LTM-training in PW (Fig. 5, black bars) with memory being tested $24 \mathrm{~h}$ later. Memory was present. We then challenged these snails with a change of context using carrot-scented water $2 \mathrm{~h}$ after the TM (Fig. 5, carrot hypoxic PW; hatched bar). As can be seen in the figure, they performed in the carrot context as naive snails; memory was not apparent (i.e., there was no statistical difference between carrot TM and TS1). Thus, context-specific memory was demonstrated. Next, we repeated this same experiment, only the snails were now trained in CE (Fig. 5, white bars). We tested for memory $24 \mathrm{~h}$ later (Fig. 5, 24 TM, white bar), and, as expected, LTM was demonstrated. We then tested for context specificity $2 \mathrm{~h}$ later in carrot-scented water (i.e., carrot TM; hatched bar) and with a different cohort of snails in PW 2 h later (Fig. 5, PW TM, black bar). These snails did not exhibit contextspecific LTM (i.e., they did not behave as naive snails in either of the new contexts). That is, the number of attempted openings in the carrot TM and standard pond water TM is significantly lower than TS1. Carrot yoked controls (striped bar) do not demon- 
strate memory. Thus, when trained in CE, snails did not demonstrate context-specific memory, that is, the memory was context generalized.

It was possible, however, that snails trained in $\mathrm{CE}$ are no longer able to detect the carrot scent. That is training in CE somehow "jams" the chemosensory apparatus, possibly preventing the carrot smell to be perceived by the snails in CE. To exclude this possibility, we used the readily observable feeding behavior of the snails. When presented with a sucrose stimulus to the lip, the snails increase their feeding behavior (Rose and Benjamin, 1979; Kemenes and Benjamin, 1989; Kawai et al., 2004). When carrot juice is applied, a similar behavior is observed. We measured the number of rasps per minute of snails in PW and then in carrotscented PW, CE, and carrot/CE, respectively. Snails in PW had a mean rasping rate of $1.64 \mathrm{rpm}$, which dramatically increased in carrot $\mathrm{PW}$ to $9.51 \mathrm{rpm}(n=24, p<0.01)$. In $\mathrm{CE}$, the mean rasping rate was $1.48 \mathrm{rpm}$, which is not significantly different from PW $(n=24, p>0.05)$. When tested in carrot/CE, the mean rasping rate increased to $9.35 \mathrm{rpm}$, which is significantly increased from CE alone $(n=24, p<0.01)$ but not significantly different from carrot PW $(n=24, p>0.05)$. No difference was found in mean rasps per minute between treatments given in the order presented here or the reverse order (data not shown). Therefore, we conclude that snails exposed to CE are capable of detecting and responding appropriately to the carrot scent. That is, CE does not jam the sensory apparatus used to detect the carrot scent.

\section{Discussion}

In biological systems, the term "stress" is described as any condition that seriously perturbs the physiological or psychological homeostasis of an organism (Kim and Diamond, 2002). Theoretically, the relationship between stress and memory formation can be described as an inverted U-shaped curve, the classic YerkesDotson 1908 curve (Shors, 2004) where, at low levels of stress, memory is impaired because of a lack of stimulation (i.e., boredom); whereas at intermediate levels of stress, memory is enhanced; and finally at high levels of stress, the organisms' ability to form memory is again reduced (Martens et al., 2007a,b). In nature, animals are exposed to a broad range of stressful challenges; of these factors, predator-prey interactions are one of the most intensely studied (Rundle and Bronmark, 2001; Herberholz et al., 2004; Stokes et al., 2004). Prey animals often exhibit a wide range of responses to predator threat, including morphological (e.g., spines and armor), physiological (e.g., toxins), and behavioral adaptations, including predator avoidance (Kavaliers and Choleris, 2001). Learning about predators is expected to have high adaptive payoffs in any species that can alter their behavior during times of predation risk (Coolen et al., 2005), and the importance of the intensity of threat has been emphasized in both laboratory and field experiments (Matsuda et al., 1993; Abrams, 1994; Lima, 1998). With respect to the Yerkes-Dotson memory curve, any predator-prey encounter in which the prey is aware of a predator presence yet escapes the interaction with its life should fall within a range close to the optimal stress intensity for memory formation and, therefore, should augment memory formation. Unfortunately, attempts to confirm this theory experimentally has yielded mixed results. In humans and rodents, although its has been shown that stressful and even traumatic events can produce strong, long-lasting memories (Cahill and McGaugh, 1998; LeDoux, 2000; Bremner, 2001), the reverse has also been demonstrated in which stress impairs memory and can even produce amnesia (Bremner et al., 1996; Payne et al., 2002; Jackson et al., 2006).

We showed here, using snails that have not experienced a natural predator for more than 250 generations that after exposure to CE, LTM formation was significantly enhanced compared with the typical memory in PW. First, LTM resulted from the ITM-training procedure, the duration of memory persistence increasing from 3 to $48 \mathrm{~h}$. Second, when we used the training procedure, which in PW results in LTM persisting for $24 \mathrm{~h}$, LTM now persisted for at least $8 \mathrm{~d}$. Thus, CE exposure significantly enhanced LTM persistence. This is not surprising considering that a single predator-prey interaction can have considerable influence on the fitness of the prey animal. Another important observation is that, when our snails were directly exposed to the predator, memory formation was not blocked. This suggests that in our model system, both predator detection and direct exposure fall within the realm of optimal memory formation and therefore provide these animals with an appropriate functional response. These results are consistent with the hypothesis that enhanced memory formation as a result of exposure to a natural predator is in itself a form of inducible anti-predator response that is elicited by the detection of a potential predator. This defense enhances the persistence of the newly formed memory and therefore possibly conveys an adaptive advantage to the snail.

Neural correlates of the CE-enhanced memory were also obtained in RPeD1. We chose to record from this neuron because it is a necessary site for LTM formation, memory reconsolidation, extinction, and forgetting (Scheibenstock et al., 2002; Sangha et al., 2003a,b; Lattal et al., 2006). Moreover, we and others (Lowe and Spencer, 2006) have shown previously that significant alterations in various electrophysiological parameters in this neuron adequately reflect the significant behavioral changes that occur after training in either intact or in initially naive semi-intact preparations. Thus, we were not too surprised to find that the enhanced LTM caused by training in CE was reflected in altered $\mathrm{RPeD} 1$ activity. Our working hypothesis is that CE exposure alters the molecular machinery in neurons (e.g., RPeD1) that are responsible for forming and maintaining the memory. As has been demonstrated previously (Syed et al., 1990, 1993; Inoue et al., 1996; Lukowiak et al., 2003b), depolarization of RPeD1 indirectly excites the VI/J motor neurons, which are directly involved in pneumostome opening during respiration at the air/water interface. It follows that a reduction in the firing frequency of $\mathrm{RPeD1}$ would necessarily lead to reduced motor neuron activation and thereby a reduction in pneumostome openings. How training while exposed to $\mathrm{CE}$ affects both protein synthesis and new gene transcription required for memory formation at the neuronal level and whether these changes are limited to the central network or extend to the peripheral network are unknown and currently under investigation. Exposure to CE only results in short-term changes in the electrophysiological properties of $\mathrm{RPeD} 1$; it is only with the combination of CE exposure and training that results in long-lasting changes in the electrophysiological properties of this neuron. These data suggest that the perception of the CE primes the molecular mechanisms required for LTM formation (Parvez et al., 2006) so that a specific training procedure is more efficacious in producing LTM. These findings recapitulate those found in many mammalian predator-stress models: (1) the snails recognize a predator odor and initiate both primary (increased exploratory/searching phase, shadow sensitivity, righting response, and surfacing) and secondary defense (full body-withdrawal response) behaviors (Covich et al., 1994; Apfelbach et al., 2005; Orr et al., 2007) according to the intensity 
of the predator-prey interaction, and (2) learning while in the presence of ecologically relevant stressor augments memory formation (Cahill, 2000; McGaugh, 2000; Shors, 2004; Joels et al., 2006).

Training in CE resulted in stimulus generalization because context-specific memory was not seen. Previously, we demonstrated that Lymnaea exhibit context-specific memory (Haney and Lukowiak, 2001); that is, when a memory is formed in a specific context (e.g., food odorant), it is only recalled in that same context. However, here we discovered that context-specific memory was not seen with exposure to CE despite the snails ability to differentiate between the different contexts (Sugai et al., 2006) and respond appropriately to the given stimuli (i.e., increased feeding behavior in a carrot stimulus). The ability to generalize a predator encounter to multiple contexts could result in both direct and indirect effects on an organism's fitness (Covich et al., 1994; Chivers and Smith, 1998; Kats and Dill, 1998; Kavaliers and Choleris, 2001; Jacobsen and Stabell, 2004). Therefore, the ability of a prey species to generalize a hazardous encounter to a broad range of situations would have direct consequences for survival. Furthermore, Rigby and Jokela (2000) have demonstrated that the withdrawal defense behavior exhibited by Lymnaea when under attack by a crayfish predator, while allowing snails to survive, may have negative effects on their immune system, increasing their susceptibility to pathogens and decreased reproduction. Therefore, the ability to generalize the context of a predator encounter to other circumstances would have further indirect adaptive value.

Together, our data suggest that the predator-augmented memory formation may help to prepare the animal to face future challenging situations, whereas the relevant information encoded as memory is deemed important enough that it will be recalled under a broader range of situations. The ecological implications of memory formation, which presumably are crucial to survival, remain hard to elucidate because of the difficulty demonstrating causal links (Bolhuis and Macphail, 2001) between ecologically relevant behaviors (Kavaliers and Choleris, 2001) and the neural substrates driving those behaviors (Fei et al., 2007). A vast amount of literature pertaining to contextual fear learning exists in mammalian systems (Vianna et al., 2004). Context learning has also been demonstrated in many invertebrates, such as the honeybee, Aplysia, cockroaches, Drosophila, and many others (Waddell and Quinn, 2001; Giurfa, 2003; Leonard and Edstrom, 2004). However, investigating the consequences of predatorprey interactions on memory formation has received little attention in these model systems. Here we can begin to form these links by investigating the molecular events in a single neuron in Lymnaea that has been shown to be necessary for this LTM formation. We can now describe how an ecologically relevant stress stimulus (predator detection) affects the physiological properties of a single neuron shown to be a necessary site of LTM formation and how operant conditioning in the presence of this predator causes memory augmentation in this neuron.

To further strengthen our knowledge about the causal links between these behaviors and the neural substrates driving them, we must answer such questions as follows: What are the molecular mechanisms of predator-augmented memory at the neuronal level? What role do both peripheral (i.e., osphradium and osphradial ganglion) and central components of the nervous system play in either predator detection or predator augmented memory formation? What chemical(s) does the predator release that are detected by the prey? And do Lymnaea populations that have not historically been subjected to crayfish predation also respond to crayfish effluent or other predator scents? These investigations are currently ongoing in our laboratory.

\section{References}

Abrams P (1994) Should prey overestimate the risk of predation. Am Naturalist 144:317-328.

Apfelbach R, Blanchard CD, Blanchard RJ, Hayes RA, McGregor IS (2005) The effects of predator odors in mammalian prey species: a review of field and laboratory studies. Neurosci Biobehav Rev 29:1123-1144.

Benjamin PR, Staras K, Kemenes G (2000) A systems approach to the cellular analysis of associative learning in the pond snail Lymnaea. Learn Mem 7:124-131.

Bolhuis JJ, Macphail EM (2001) A critique of the neuroecology of learning and memory. Trends Cogn Sci 5:426-433.

Bremner JD (2001) Hypotheses and controversies related to effects of stress on the hippocampus: an argument for stress-induced damage to the hippocampus in patients with posttraumatic stress disorder. Hippocampus 11:75-81; discussion 82-74.

Bremner JD, Krystal JH, Charney DS, Southwick SM (1996) Neural mechanisms in dissociative amnesia for childhood abuse: relevance to the current controversy surrounding the "false memory syndrome". Am J Psychiatry 153:71-82.

Burks RL, Lodge DM (2002) Cued in: advances and opportunities in freshwater chemical ecology. J Chem Ecol 28:1901-1917.

Cahill L (2000) Neurobiological mechanisms of emotionally influenced, long-term memory. Prog Brain Res 126:29-37.

Cahill L, McGaugh JL (1998) Mechanisms of emotional arousal and lasting declarative memory. Trends Neurosci 21:294-299.

Chivers DP, Smith RJF (1998) Chemical alarm signalling in aquatic predator-prey systems: a review and prospectus. Ecoscience 5:338-352.

Coolen I, Dangles O, Casas J (2005) Social learning in noncolonial insects? Curr Biol 15:1931-1935.

Covich P, Crowl T, Alexander JE, Vaughn CC (1994) Predator-avoidance responses in fresh-water decapod-gastropod interactions mediated by chemical stimuli. J North Am Benthol Soc 13:283-290.

Dalesman S, Rundle SD, Coleman RA, Cotton PA (2006) Cue association and antipredator behaviour in a pulmonate snail, Lymnaea stagnalis Anim Behav 71:789-797.

de Kloet ER, Oitzl MS, Joels M (1999) Stress and cognition: are corticosteroids good or bad guys? Trends Neurosci 22:422-426.

Fei G, Guo C, Sun H-S, Feng Z-P (2007) Chronic hypoxia stress-induced differential modulation of heat-shock protein 70 and presynaptic proteins. J Neurochem 100:50-61.

Giurfa M (2003) Cognitive neuroethology: dissecting non-elemental learning in a honeybee brain. Curr Opin Neurobiol 13:726-735.

Haney J, Lukowiak K (2001) Context learning and the effect of context on memory retrieval in Lymnaea. Learn Mem 8:35-43.

Herberholz J, Sen MM, Edwards DH (2004) Escape behavior and escape circuit activation in juvenile crayfish during prey-predator interactions. J Exp Biol 207:1855-1863.

Inoue T, Takasaki M, Lukowiak K, Syed N (1996) Inhibition of the respiratory pattern-generating neurons by an identified whole-body withdrawal interneuron of Lymnaea stagnalis. J Exp Biol 199:1887-1898.

Inoue T, Haque Z, Lukowiak K, Syed NI (2001) Hypoxia-induced respiratory patterned activity in Lymnaea originates at the periphery. J Neurophysiol 86:156-163.

Jackson ED, Payne JD, Nadel L, Jacobs WJ (2006) Stress differentially modulates fear conditioning in healthy men and women. Biol Psychiatry 59:516-522.

Jacobsen HP, Stabell OB (2004) Antipredator behaviour mediated by chemical cues: the role of conspecific alarm signalling and predator labelling in the avoidance response of a marine gastropod. Oikos 104:43-50.

Joels M, Pu Z, Wiegert O, Oitzl MS, Krugers HJ (2006) Learning under stress: how does it work? Trends Cogn Sci 10:152-158.

Kats LB, Dill LM (1998) The scent of death: chemosensory assessment of predation risk by prey animals. Ecoscience 5:361-394.

Kavaliers M, Choleris E (2001) Antipredator responses and defensive behavior: ecological and ethological approaches for the neurosciences. Neurosci Biobehav Rev 25:577-586.

Kawai R, Sunada H, Horikoshi T, Sakakibara M (2004) Conditioned taste aversion with sucrose and tactile stimuli in the pond snail Lymnaea stagnalis. Neurobiol Learn Mem 82:164-168. 
Kemenes G, Benjamin PR (1989) Appetitive learning in snails shows characteristics of conditioning in vertebrates. Brain Res 489:163-166.

Kim JJ, Diamond DM (2002) The stressed hippocampus, synaptic plasticity and lost memories. Nat Rev Neurosci 3:453-462.

Lattal KM, Radulovic J, Lukowiak K (2006) Extinction: does it or doesn't it? The requirement of altered gene activity and new protein synthesis. Biol Psychiatry 60:344-351.

LeDoux JE (2000) Emotion circuits in the brain. Annu Rev Neurosci 23:155-184.

Leonard JL, Edstrom JP (2004) Parallel processing in an identified neural circuit: the Aplysia californica gill-withdrawal response model system. Biol Rev Camb Philos Soc 79:1-59.

Lima SL (1998) Stress and decision making under the risk of predation: Recent developments from behavioral, reproductive, and ecological perspectives. Stress Behav 27:215-290.

Lowe MR, Spencer GE (2006) Perturbation of the activity of a single identified neuron affects long-term memory formation in a molluscan semiintact preparation. J Exp Biol 209:711-721.

Lukowiak K, Ringseis E, Spencer G, Wildering W, Syed N (1996) Operant conditioning of aerial respiratory behaviour in Lymnaea stagnalis. J Exp Biol 199:683-691.

Lukowiak K, Cotter R, Westly J, Ringseis E, Spencer G (1998) Long-term memory of an operantly conditioned respiratory behaviour pattern in Lymnaea stagnalis. J Exp Biol 201:877-882.

Lukowiak K, Adatia N, Krygier D, Syed N (2000) Operant conditioning in Lymnaea: evidence for intermediate- and long-term memory. Learn Mem 7:140-150.

Lukowiak K, Sangha S, McComb C, Varshney N, Rosenegger D, Sadamoto H, Scheibenstock A (2003a) Associative learning and memory in Lymnaea stagnalis: how well do they remember? J Exp Biol 206:2097-2103.

Lukowiak K, Sangha S, Scheibenstock A, Parvez K, McComb C, Rosenegger D, Varshney N, Sadamoto H (2003b) A molluscan model system in the search for the engram. J Physiol (Paris) 97:69-76.

Martens K, Amarell M, Parvez K, Hittel K, De Caigny P, Ito E, Lukowiak K (2007a) One-trial conditioning of aerial respiratory behaviour in Lymnaea stagnalis. Neurobiol Learn Mem 88:232-242.

Martens KR, De Caigny P, Parvez K, Amarell M, Wong C, Lukowiak K (2007b) Stressful stimuli modulate memory formation in Lymnaea stagnalis. Neurobiol Learn Mem 87:391-403.

Matsuda H, Abrams Pa, Hori H (1993) The effect of adaptive antipredator behavior on exploitative competition and mutualism between predators. Oikos 68:549-559.

McComb C, Meems R, Syed N, Lukowiak K (2003) Electrophysiological differences in the CPG aerial respiratory behavior between juvenile and adult Lymnaea. J Neurophysiol 90:983-992.

McComb C, Varshney N, Lukowiak K (2005) Juvenile Lymnaea ventilate, learn and remember differently than do adult Lymnaea. J Exp Biol 208:1459-1467.

McGaugh JL (2000) Memory-a century of consolidation. Science 287:248-251.

Orr MV, El-Bekai M, Lui M, Watson K, Lukowiak K (2007) Predator detection in Lymnaea stagnalis. J Exp Biol 210:4150-4158.
Parvez K, Stewart O, Sangha S, Lukowiak K (2005) Boosting intermediateterm into long-term memory. J Exp Biol 208:1525-1536.

Parvez K, Rosenegger D, Martens K, Orr M, Lukowiak K (2006) Learning at a snail's pace. Can J Neurol Sci 33:347-356.

Payne JD, Nadel L, Allen JJ, Thomas KG, Jacobs WJ (2002) The effects of experimentally induced stress on false recognition. Memory 10:1-6.

Rigby MC, Jokela J (2000) Predator avoidance and immune defence: costs and trade-offs in snails. Proc Biol Sci 267:171-176.

Rose RM, Benjamin PR (1979) The relationship of the central motor pattern to the feeding cycle of Lymnaea stagnalis. J Exp Biol 80:137-163.

Rundle SD, Bronmark C (2001) Inter- and intraspecific trait compensation of defence mechanisms in freshwater snails. Proc R Soc Lond B Biol Sci 268:1463-1468.

Sangha S, Scheibenstock A, Lukowiak K (2003a) Reconsolidation of a longterm memory in Lymnaea requires new protein and RNA synthesis and the soma of right pedal dorsal 1. J Neurosci 23:8034-8040.

Sangha S, Scheibenstock A, Morrow R, Lukowiak K (2003b) Extinction requires new RNA and protein synthesis and the soma of the cell right pedal dorsal 1 in Lymnaea stagnalis. J Neurosci 23:9842-9851.

Scheibenstock A, Krygier D, Haque Z, Syed N, Lukowiak K (2002) The Soma of RPeD1 must be present for long-term memory formation of associative learning in Lymnaea. J Neurophysiol 88:1584-1591.

Shors TJ (2004) Learning during stressful times. Learn Mem 11:137-144.

Spencer GE, Syed NI, Lukowiak K (1999) Neural changes after operant conditioning of the aerial respiratory behavior in Lymnaea stagnalis. J Neurosci 19:1836-1843.

Spencer GE, Kazmi MH, Syed NI, Lukowiak K (2002) Changes in the activity of a CPG neuron after the reinforcement of an operantly conditioned behavior in Lymnaea. J Neurophysiol 88:1915-1923.

Stokes VL, Pech RP, Banks PB, Arthur AD (2004) Foraging behaviour and habitat use by Antechinus flavipes and Sminthopsis murina (Marsupialia: Dasyuridae) in response to predation risk in eucalypt woodland. Biol Conserv 117:331-342.

Sugai R, Shiga H, Azami S, Watanabe T, Sadamoto H, Fujito Y, Lukowiak K, Ito $\mathrm{E}$ (2006) Taste discrimination in conditioned taste aversion of the pond snail Lymnaea stagnalis. J Exp Biol 209:826-833.

Syed NI, Bulloch AG, Lukowiak K (1990) In vitro reconstruction of the respiratory central pattern generator of the mollusk Lymnaea. Science 250:282-285.

Syed NI, Ridgway RL, Lukowiak K, Bulloch AG (1992) Transplantation and functional integration of an identified respiratory interneuron in Lymnaea stagnalis. Neuron 8:767-774.

Syed NI, Roger I, Ridgway RL, Bauce LG, Lukowiak K, Bulloch AG (1993) Identification, characterisation and in vitro reconstruction of an interneuronal network of the snail Helisoma trivolvis. J Exp Biol 174:19-44.

Taylor BE, Lukowiak K (2000) The respiratory central pattern generator of Lymnaea: a model, measured and malleable. Respir Physiol 122:197-207.

Vianna MR, Coitinho AS, Izquierdo I (2004) Role of the hippocampus and amygdala in the extinction of fear-motivated learning. Curr Neurovasc Res 1:55-60.

Waddell S, Quinn WG (2001) What can we teach Drosophila? What can they teach us? Trends Genet 17:719-726. 